\title{
Effect of evolocumab on the progression and stability of atherosclerotic plaques as evaluated by grayscale and iMAP-IVUS
}

\author{
Qingzan Kong ${ }^{1}$, Miao Liu ${ }^{1}$, Yueyan $\mathrm{Li}^{1}$, Qing $\mathrm{Zhu}^{2}$, Guohai Su${ }^{1}$ \\ ${ }^{1}$ Department of Cardiology, Jinan Central Hospital, Cheeloo College of Medicine, Shandong University, Jinan, China; ${ }^{2}$ Department of Pathology, \\ Blood Center of Shandong Province, Jinan, China \\ Contributions: (I) Conception and design: G Su; (II) Administrative support: Q Zhu, G Su; (III) Provision of study materials or patients: Q Kong; (IV) \\ Collection and assembly of data: Q Kong, M Liu; (V) Data analysis and interpretation: Q Kong, Y Li; (VI) Manuscript writing: All authors; (VII) \\ Final approval of manuscript: All authors. \\ Correspondence to: Guohai Su. Department of Cardiology, Jinan Central Hospital, Cheeloo College of Medicine, Shandong University, No. 105, \\ Jiefang Road, Lixia District, Jinan 250013, China. Email: guohaisu1234@163.com.
}

Background: Evolocumab inhibits the proprotein convertase subtilisin/kexin type 9 protein and is a potent cholesterol-lowering drug. However, the relationship between evolocumab and inflammation, and the effects of evolocumab on the stability of atherosclerotic plaques remain unknown.

Methods: Twenty-seven purebred New Zealand rabbits were fed with an atherogenic diet for 2 weeks. The abdominal aortic endothelium was balloon-injured. The rabbits were divided into the atorvastatin ( $2 \mathrm{mg} / \mathrm{kg} /$ day; Ato), evolocumab (7 mg/kg/2 weeks, Evo) and control groups. Intravascular ultrasound (IVUS) images of the abdominal artery were analyzed at 10 and 18 weeks. Additionally, the serum levels of the biomarkers were measured at baseline, and at 10 and 18 weeks.

Results: The serum levels of triglycerides, total cholesterol, low-density lipoprotein cholesterol (LDL-C), and monocyte chemoattractant protein-1 (MCP-1) increased after 10 weeks of administration of the proatherosclerotic diet, while the levels of high-density lipoprotein cholesterol (HDL-C) and transforming growth factor- $\beta$ (TGF- $\beta$ ) decreased. The reduction in the serum levels of triglycerides, total cholesterol, LDL-C, MCP-1, TGF- $\beta$, and toll-like receptor 4 (TLR4) following treatment with evolocumab was higher than that of atorvastatin. Both evolocumab and atorvastatin reduced the percent atheroma volume. Evolocumab increased the fibrotic\% and decreased the necrotic\%. Correlation analysis revealed that the levels of triglycerides, total cholesterol, LDL-C, MCP-1, TGF- $\beta$, and TLR4 were negatively correlated with the fibrotic\%, but were positively correlated with the necrotic\%. Multivariate linear regression analysis revealed that treatment with atorvastatin, and especially evolocumab, was a consistent predictor of the percent atheroma volume, and fibrotic and necrotic composition.

Conclusions: Proprotein convertase subtilisin/kexin type 9 regulates the serum levels of lipid and cholesterol may via inflammatory pathways. The results also indicate that evolocumab is more potent than atorvastatin in suppressing the progression and stability of atherosclerotic plaque in rabbits.

Keywords: Atherosclerosis; evolocumab; iMAP; intravascular ultrasound (IVUS)

Submitted Mar 18, 2020. Accepted for publication Aug 10, 2020.

doi: 10.21037/apm-20-690

View this article at: http://dx.doi.org/10.21037/apm-20-690 


\section{Introduction}

Atherosclerosis is a common pathological phenomenon observed in several cardiovascular diseases, and is a serious threat to human health (1). It is characterized by the massive accumulation of lipids and inflammation (2,3). However, the pathophysiology of atherosclerosis is a complicated one. Multiple factors are thought to be involved in the induction of atherosclerosis, including cholesterol, lowdensity lipoprotein cholesterol (LDL-C), and certain inflammation cytokines (4). Therefore, the reduction of lipid accumulation and suppression of inflammation may serve as crucial strategies in the prevention and treatment of atherosclerosis.

The onset of atherosclerosis is marked by chronic inflammation, which is caused by the imbalance of lipid metabolism. This subsequently results in the accumulation of cellular cholesterol, which stimulates the adaptive immune response. The chronic inflammation induces the formation of atherosclerotic plaques in the vascular tissues. The formation of atherosclerotic plaques is primarily triggered by lipid accumulation and the secretion of inflammatory cytokines. It has been reported that the considerable accumulation of LDL-C can promote atherosclerosis by inducing the formation of atherosclerotic plaques in the media intima of the arteries. On the contrary, high-density lipoprotein cholesterol (HDL-C) inhibits atherosclerosis by transporting cholesterol into the liver, and thereby reduces the cellular cholesterol content (5). Monocyte chemoattractant protein-1 (MCP-1), also known as CC-chemokine ligand 2, is actively involved in the initiation and progression of atherosclerosis (6-8). Increasing evidence suggests that the expression of MCP1 is up-regulated in patients diagnosed with atherosclerosis (9-11). Transforming growth factor- $\beta$ (TGF- $\beta$ ) is an important anti-inflammatory cytokine with pleiotropic functions (12). TGF- $\beta$ is known to control calcification and the immune response, both of which are major components of the atherosclerotic process $(13,14)$. Toll-like receptors (TLRs) are pattern recognition receptors that play a critical role in regulating the inflammatory response and in maintaining inflammatory homeostasis. Toll-like receptor 4 (TLR4) has been implicated in the pathophysiology of atherosclerosis (15) and is over-expressed in both human and murine atherosclerotic lesions at different stages of atherogenesis (16-20).

The atherosclerotic plaques can change from a stable state to an unstable state, undergo erosion and finally rupture, leading to thrombosis. From a clinical viewpoint, it is therefore crucial to explore the strategies for stabilizing atherosclerotic plaques. Several methods, including histopathological examination $(21,22)$, grayscale intravascular ultrasound (IVUS) (23), and intravascular ultrasound elastography (IVUSE) (24) have been previously employed in animal studies for studying the stability and progression of atherosclerotic plaques. However, only a few animal studies have used color IVUS form to determine the stability and progression of atherosclerotic plaques. The iMAP (Boston Scientific, MA, USA) color IVUS systems has a relatively new software package for analysis of the fibrotic, lipidic, necrotic, calcific plaque components of atherosclerotic plaques on the basis of the backscattered ultrasound frequency spectrum $(25,26)$. The feasibility of using IMAP-IVUS in diagnosis of plaque vulnerability and prediction of plaque components in patients with acute coronary syndrome has been confirmed $(27,28)$.

Evolocumab is an inhibitor of the proprotein convertase subtilisin/kevin type 9 (PCSK9) protein. It has been reported that $\mathrm{PCSK} 9$ promotes atherosclerosis by increasing the serum levels of LDL-C (29). The inhibition of PCSK9 has been shown to significantly reduce the plasma levels of LDL-C in atherosclerotic patients, thus reducing the incidence and mortality of cardiovascular diseases (30-32). In this study, we investigated the relationship between inflammation and evolocumab, and the effects of evolocumab on the serum levels of lipids and the plaque components in the abdominal arteries of a rabbit model of atherosclerosis using iMAP-IVUS.

We present the following article in accordance with the ARRIVE reporting checklist (available at http://dx.doi. org/10.21037/apm-20-690).

\section{Methods}

\section{Experimental animals}

Twenty-seven 4-month-old New Zealand white male rabbits (mean weight $1.84 \pm 0.13 \mathrm{~kg}$ ) were provided by the Jinan Xilingjiao Culture and Breeding Center, Jinan, China (license number: SCXK(LU) 20160005). The animals were fed with an atherogenic diet containing $1 \%$ cholesterol and $99 \%$ normal rabbit diet $(150 \mathrm{~g} /$ day $)$ for 2 weeks. The abdominal aortic endothelium of all the rabbits was ballooninjured after 2 weeks of administration of the atherogenic diet, as described in previous studies (33-35). At the end of week 10, the rabbits were randomly divided into three 
groups: the Ato group ( $\mathrm{n}=9)$, in which the rabbits received intragastric administration of $2 \mathrm{mg} / \mathrm{kg}$ atorvastatin calcium (Pfizer, NY, USA) through a stomach tube once a day for 8 weeks; the Evo group ( $\mathrm{n}=9$ ), in which $7 \mathrm{mg} / \mathrm{kg}$ evolocumab (Amgen Inc., Thousand Oaks, CA, USA) was administered biweekly via subcutaneous injections; and the control group in which the animals received normal saline. The dosage of atorvastatin and evolocumab was determined according to the body surface area and body weights of humans and animals. Doses of $2 \mathrm{mg} / \mathrm{kg}$ atorvastatin calcium and $7 \mathrm{mg} / \mathrm{kg}$ evolocumab in rabbits were equivalent to doses of $40 \mathrm{mg}$ atorvastatin calcium and $140 \mathrm{mg}$ evolocumab in humans, respectively.

Experiments were performed under a project license (No. 2016061) granted by the ethics committee of Jinan Central Hospital Affiliated to Shandong University, in compliance with the Guide for the Care and Use of Laboratory Animals published by the US National Institutes of Health (NIH Publication No. 85-23, revised 2011). All the aseptic operations were performed under general anesthesia to minimize the suffering of the rabbits.

\section{Plasma assays}

Blood was drawn from the marginal ear vein of the rabbits that had been starved overnight for $12 \mathrm{~h}$. The blood samples were collected at baseline (beginning of the study) and at weeks 10 and 18 . The supernatant was collected after centrifugation at 4,000 rpm for $8 \mathrm{~min}$ on a TGL-16B high speed centrifuge (Anke Science Instrument Factory, Shanghai, China) and stored at $-80{ }^{\circ} \mathrm{C}$. The serum levels of triglycerides (TG), total cholesterol (TC), HDL-C, and LDL-C were measured using a biochemical autoanalyzer (Cobas 8000 c701; Roche Diagnostics, Mannheim, Germany). The levels of inflammatory factors including MCP-1 and TGF- $\beta$, were determined by enzyme-linked immunosorbent assay.

\section{Immunobistochemical staining}

All the rabbits were euthanized at the end of the study, and their abdominal aortas $(5 \mathrm{~cm}$ long proximal to the iliac arterial bifurcation) were harvested. The abdominal aortas were fixed in $4 \%$ formaldehyde and serially cut into 5 -mm-thick segments. The segments were embedded in paraffin and dehydration. The segments were subsequently sliced into $4-\mu \mathrm{m}$ thick sections, which were immunohistochemically stained with a murine antibody against rabbit TLR4 (cat. no. ab22048; Abcam, Cambridge, MA, USA) using a diaminobenzidine kit (Zhongshan Golden bridge Biotechnology, Beijing, China).

The histological images were captures with an optical microscope (Olympus, Tokyo, Japan) and the images were analyzed using an image analysis system (Image Pro Plus 6.0, Media Cybernetics Inc, MD, USA). Ten high-power fields (400x) were randomly selected from each section, and five sections were selected from each animal for measurement. The level of TLR4 was expressed in terms of the integrated optical density (IOD).

\section{IVUS measurement}

At the end of 10 and 18 weeks, the plaque volume and composition of the target segments of the abdominal aorta (10-mm-long segments with minimal luminal crosssectional area, devoid of side branches) were determined by IVUS. A 2.5-F, 40-MHz imaging catheter (Atlantis SR Pro2 and iLab Ultrasound Imaging System, Boston Scientific, Natick, MA, USA) was inserted into the abdominal aorta via the right femoral artery. The transducer of the catheter was positioned on the left renal artery ostium and withdrawn at a speed of $0.5 \mathrm{~mm} / \mathrm{s}$. The images obtained by IVUS were recorded on a DVD for further offline analyses. The bifurcation of the iliac artery was used as the reproducible landmark to synchronize the target segment at both time points (end of 10 and 18 weeks).

The data obtained by IVUS was processed according to the methods described in published guidelines (36) using special software (echoPlaque version 4.0, Indec Systems, CA, USA). The percent atheroma volume (PAV) was calculated as described in a previous study (37). PAV is defined as the percentage of vessel volume that is occupied by a plaque in the target segment. The following formula was used for calculating the PAV:

$$
\mathrm{PAV}=\frac{\sum(\text { EEMcsa }- \text { LUMENcsa })}{\sum \text { EEMcsa }} \times 100
$$

where, the abbreviations EEM and csa stand for external elastic membrane and cross-sectional area, respectively. The iMAP-IVUS radio frequency imaging system was used to characterize the tissues. The imaging system uses a pattern-recognition algorithm to decipher the spectra obtained from the Fast Fourier transformation of a human autopsy database of coronary atheroma (26). The iMAP system uses color-coding and depicts fibrotic tissues in light green, lipidic tissues in yellow, necrotic tissues in 
pink, and calcified tissues in blue. The fibrotic\%, lipidic\%, necrotic $\%$, and calcified $\%$ represent the percentages of the total volume of the atheroma in the target segment that is occupied by fibrous, lipidic, necrotic, and calcified components, respectively. The following formulae were used for calculating the fibrotic $\%$, lipidic $\%$, necrotic $\%$, and calcified $\%$ :

$$
\begin{aligned}
& \text { Fibrotic } \%=\frac{\text { Fibrotic volume }}{\text { Total atheroma volume }} \times 100 \\
& \text { Lipidic } \%=\frac{\text { Lipidic volume }}{\text { Total atheroma volume }} \times 100 \\
& \text { Necrotic } \%=\frac{\text { Necrotic volume }}{\text { Total atheroma volume }} \times 100 \\
& \text { Calcified } \%=\frac{\text { Calcified volume }}{\text { Total atheroma volume }} \times 100
\end{aligned}
$$

\section{Statistical analysis}

The continuous variables are presented as the mean \pm standard deviation for the normally distributed variables and median values (interquartile range) of the nonparametric data. The differences in the paired continuous variables between the groups were estimated by the paired sample $t$-test. One-way analysis of variance was performed to compare the differences between the groups when the homogeneity of the variance assumptions was satisfied. In other cases, the Kruskal-Wallis $\mathrm{H}$ test was applied. The Tukey's test or the Dunn-Bonferroni test was used for multiple post-hoc comparisons. The Spearman's correlation and multivariate linear regression analyses were performed to assess the relationships between the IVUS-derived parameters and the serum levels of lipids, and biomarkers. All the data were analyzed using SPSS version 22.0 (IBM, NY, USA). For the two-tailed tests, $\mathrm{P}<0.05$ was considered to be statistically significant.

\section{Results}

\section{Evolocumab decreases lipid profiles in atherosclerotic rabbits}

After 10 weeks of administration of the atherogenic diet, the serum levels of TG, TC and LDL-C increased markedly, while the levels of HDL-C decreased in the serum (Figure $1 A, B, C, D$ ). After 8 weeks of treatment with evolocumab, the serum levels of TG, TC, and LDL-C significantly decreased, while the serum levels of HDL-C significantly increased (Figure $1 E$ ). In this study, we used atorvastatin as the positive control. Atorvastatin belongs to the statin class of drugs, and is used to lower the lipid levels in the serum. The results demonstrated that evolocumab was more effective in reducing the serum levels of TG, TC, and LDL-C, than atorvastatin (Figure 1E), but there was no difference in the serum levels of HDL-C following treatment with either evolocumab or atorvastatin.

\section{Evolocumab reduces inflammation in atherosclerotic rabbits}

After 10 weeks of administration of the atherogenic diet it was observed that the serum levels of MCP-1 significantly increased, while the levels of TGF- $\beta$ significantly decreased (Figures $2 A, B)$. The administration of evolocumab and atorvastatin significantly reversed these changes; however, evolocumab was more effective at reducing the serum levels of MCP-1 and increasing the levels of TGF- $\beta$ compared to the efficacy exhibited by atorvastatin (Figure $2 C$ ). Additionally, the results of immunohistochemical staining assays revealed that both evolocumab and atorvastatin significantly reduced the expression of the TLR4 protein (Figures 2D,E). The expression of the TLR4 protein in the abdominal aorta of atherosclerotic rabbits that received evolocumab was significantly lower than that of the rabbits that were treated with atorvastatin (Figure 2E).

\section{Grayscale and iMAP-IVUS volumetric parameters}

After 8 weeks of treatment with atorvastatin and evolocumab, the PAV of the atherosclerotic rabbits in the treatment groups decreased significantly (Figure 3A). The fibrotic\% increased significantly (Figure $3 B$ ) while the necrotic\% decreased (Figure 3C), compared to those of the control group at the end of 18 weeks. The necrotic\% of the atorvastatin and evolocumab groups was significantly lower than that of the atherosclerotic rabbits in the control group at the end of 10 weeks. There were no differences in the lipidic\% and calcified $\%$ in the abdominal aorta (Figure $3 D, E)$. The representative grayscale and corresponding iMAP-IVUS images are illustrated in Figure S1. 

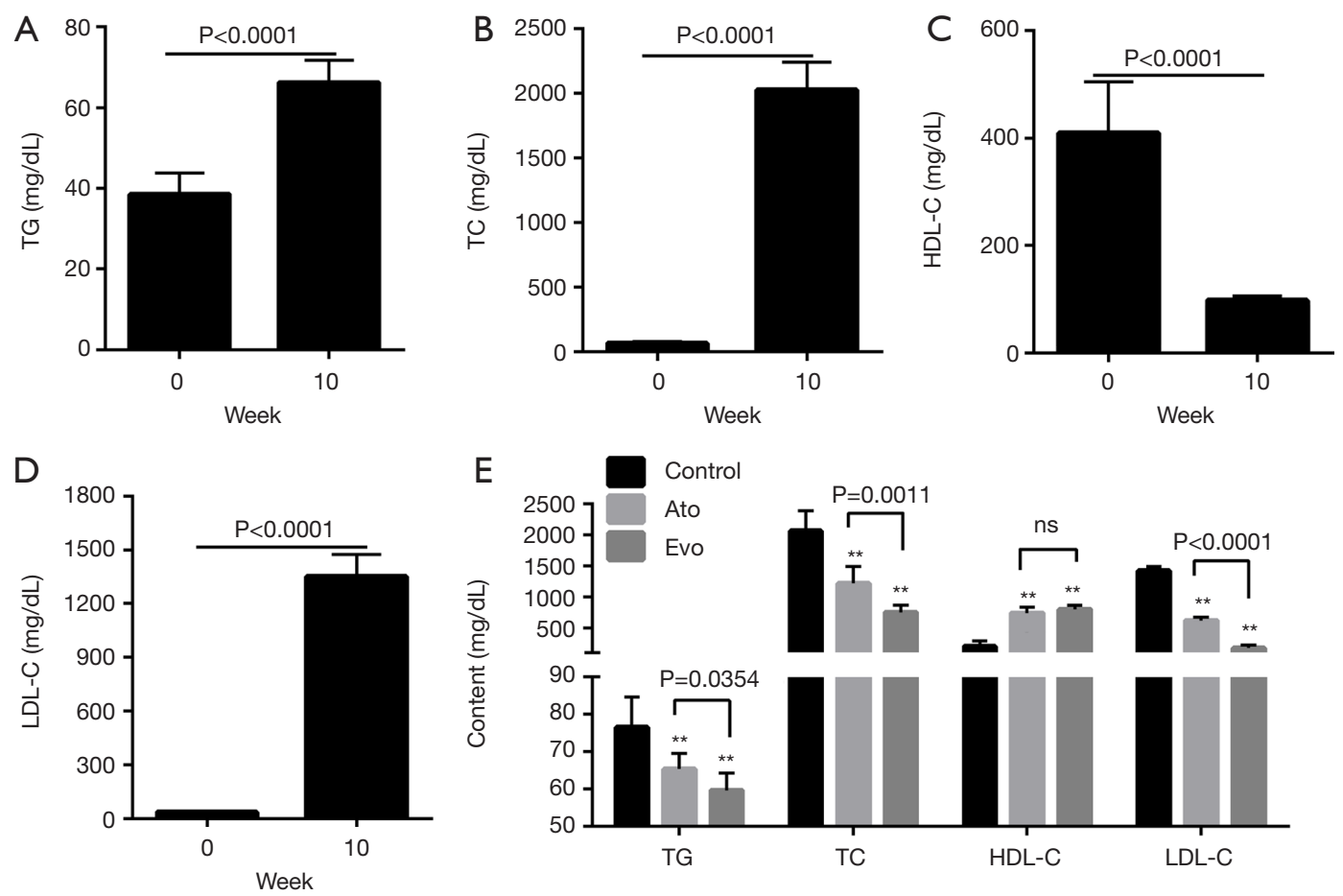

Figure 1 Effect of the atherogenic diet and treatment with atorvastatin and evolocumab on the serum lipid profiles. HDL-C, high-density lipoprotein cholesterol; LDL-C, low-density lipoprotein cholesterol; TC, total cholesterol; TG, triglyceride; NS, non-significant (P $\geq 0.05$ ). **, $\mathrm{P}<0.001$ vs. control.

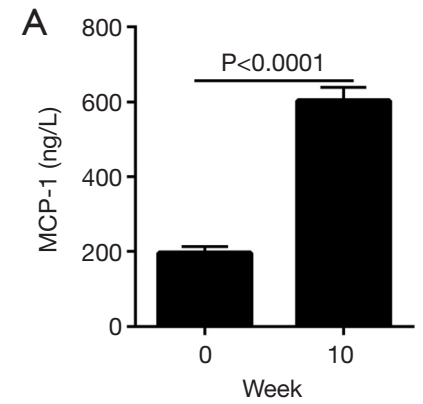

D

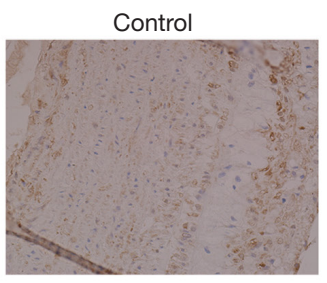

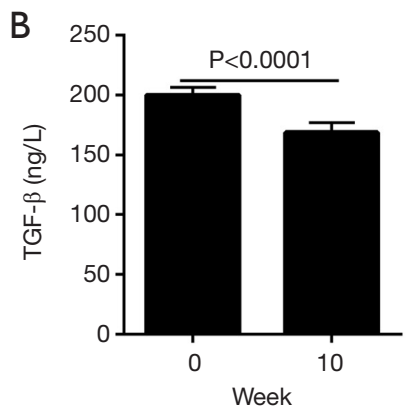

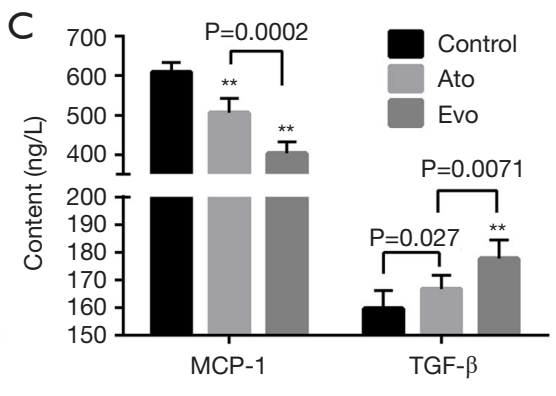

Ato

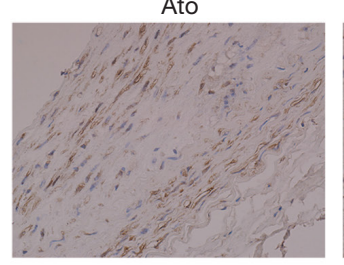

Evo

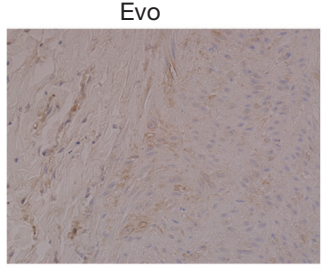

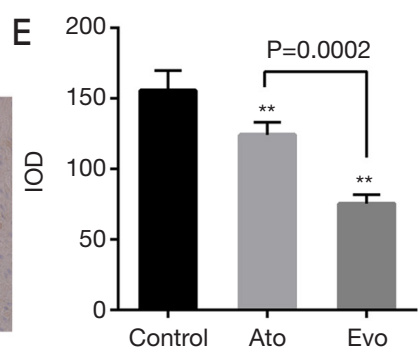

Figure 2 Response of inflammatory biomarkers and TLR4 to the atherogenic diet and treatments with atorvastatin and evolocumab. (A,B) Serum levels of MCP-1 and TGF- $\beta$, respectively, at the baseline and at week 10; (C) the comparison of the serum levels of MCP-1 and TGF- $\beta$ levels across the three experimental groups at the 18 weeks; (D) the representative images obtained from the immunochemistry assay for the TLR4 protein (400x); (E) the comparison of the integrated optical density (IOD) values for the TLR4 protein. NS, non-significant $(\mathrm{P} \geq 0.05)$. **, $\mathrm{P}<0.001$ vs. control. 

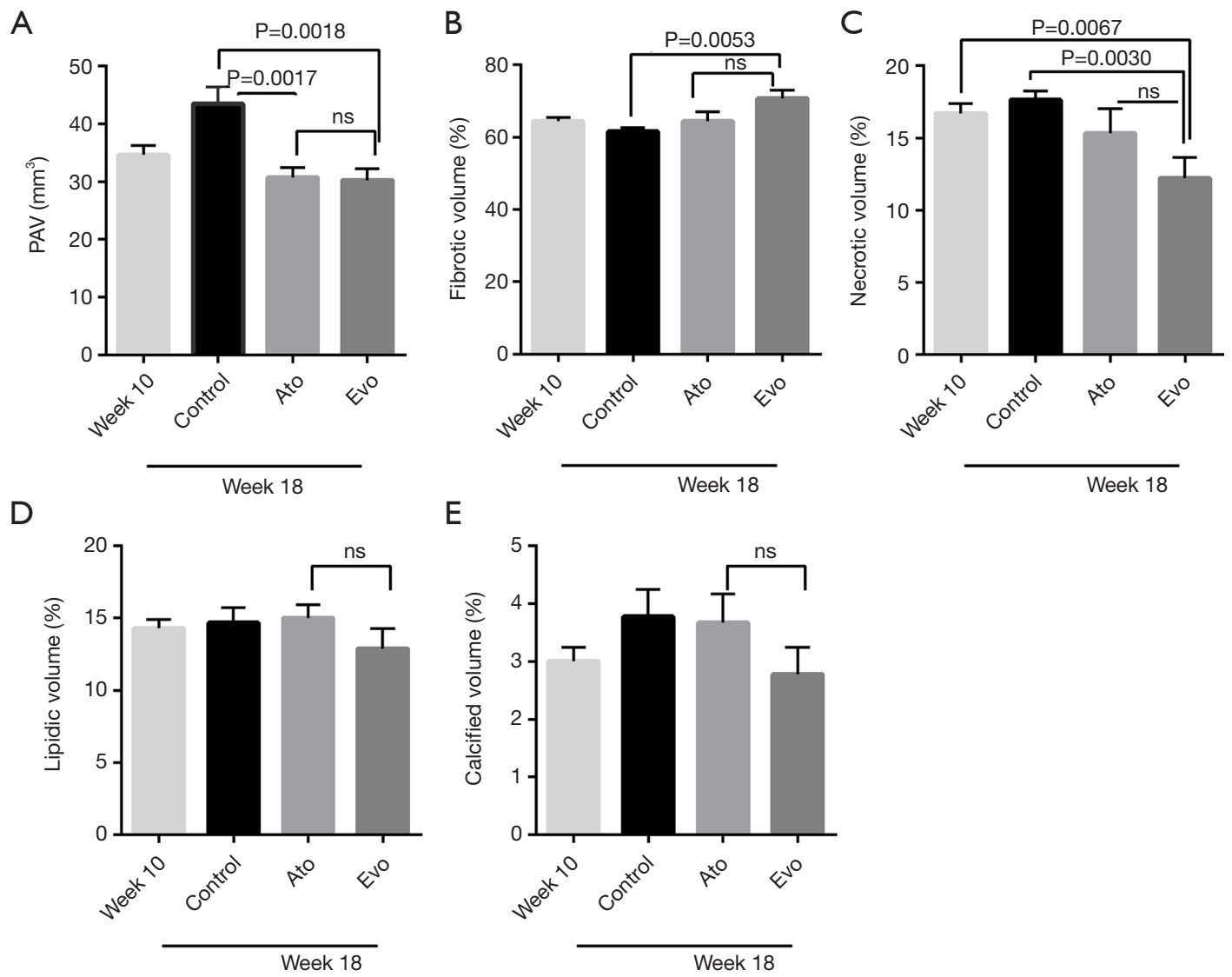

Figure 3 Effect of the atherogenic diet and treatments with atorvastatin and evolocumab on the atherosclerotic plaques. (A) The percent atheroma volume (PAV) in abdominal aorta; (B,C,D,E) the fibrotic\%, necrotic\%, lipidic\%, and calcified\% in the abdominal aorta of the rabbits, respectively. NS, non-significant $(\mathrm{P} \geq 0.05)$.

\section{Bivariate correlations analysis and multivariate linear regression analysis}

Figure 4 depicts the relationships between PAV and the biomarkers at 18 weeks. The values of PAV were found to be positively correlated with the serum levels of TG $(r=0.596, P=0.001)$, TC $(r=0.549, P=0.003)$, LDL-C $(\mathrm{r}=0.560, \mathrm{P}=0.002), \mathrm{MCP}-1(\mathrm{r}=0.575, \mathrm{P}=0.002)$, and TLR4 expression $(\mathrm{r}=0.511, \mathrm{P}=0.006)$, while it was negatively correlated with the levels of TGF- $\beta$ ( $r=-0.437, \mathrm{P}=0.023)$. Correlation analyses (Table 1) revealed that the fibrotic\% was negatively correlated with the TC $(r=-0.696, \mathrm{P}<0.001)$, TG $(\mathrm{r}=-0.385, \mathrm{P}=0.048), \mathrm{LDL}-\mathrm{C}(\mathrm{r}=-0.651, \mathrm{P}<0.001)$, calcified \% ( $r=-0.603, \mathrm{P}=0.001), \mathrm{MCP}-1(\mathrm{r}=-0.412$, $\mathrm{P}=0.033)$ and TLR4 ( $\mathrm{r}=-0.463, \mathrm{P}=0.015)$ at the end of 18 weeks. Fibrotic\% was found to be positively correlated with the expression of TGF- $\beta$ at 18 weeks $(r=0.396, P=0.041)$. However, necrotic\% was found to be positively correlated with TC $(\mathrm{r}=0.793, \mathrm{P}<0.001)$, TG $(\mathrm{r}=0.556, \mathrm{P}=0.003)$, LDL-C ( $\mathrm{r}=0.751, \mathrm{P}<0.001)$, calcified\% $(\mathrm{r}=0.517, \mathrm{P}=0.006)$, MCP-1 ( $\mathrm{r}=0.457, \mathrm{P}=0.016)$, and TLR4 expression $(\mathrm{r}=0.493$, $\mathrm{P}=0.009)$. Necrotic\% was found to be negatively correlated with the expression of TGF- $\beta(r=-0.453, \mathrm{P}=0.018)$.

A multivariate analysis was performed using the variables derived from IVUS, namely, PAV, fibrotic\%, and necrotic\%, using the following candidate predictors: the different treatments (converting to two dummy variables: Ato and Evo), TG, TC, HDL-C, LDL-C, MCP-1, TGF- $\beta$, and TLR4. The values of all the aforementioned variables represented the follow-up values for week 18 . It is evident from Table 2 that the type of treatment was the consistent predictor for the variables derived from IVUS, and evolocumab had higher absolute values of regression coefficients compared to those of atorvastatin (34.46 vs. $21.007,12.326$ vs. 2.509, and 7.753 vs. 2.133, for evolocumab $v s$. atorvastatin, respectively). It was additionally 

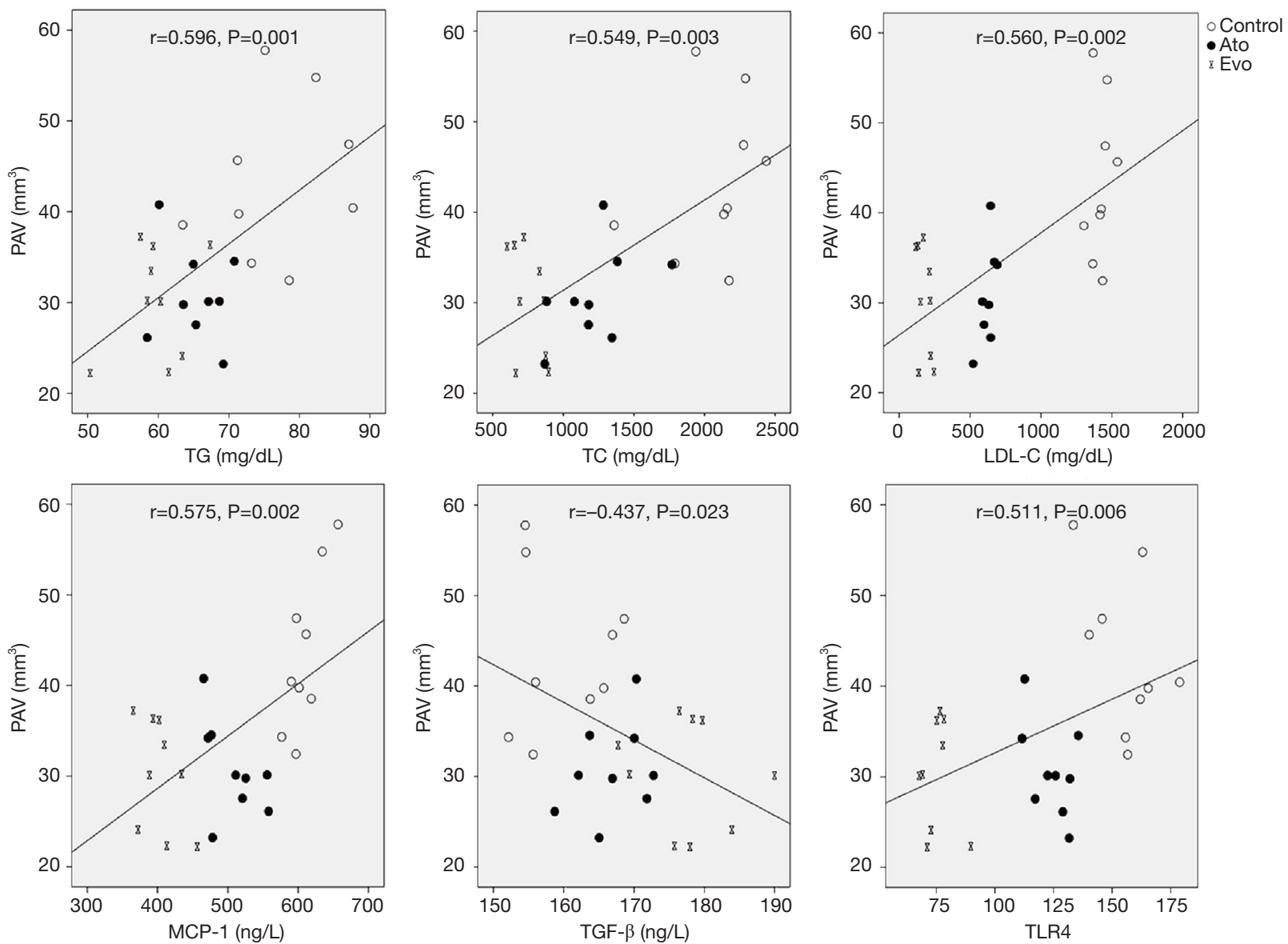

Figure 4 The variables intravascular ultrasound (IVUS) had significant positive correlation with the percent atheroma volume (PAV).

Table 1 Relationships between fibrotic and necrotic volume and other biomarkers at week 18

\begin{tabular}{|c|c|c|c|c|c|c|c|}
\hline Variables & $\mathrm{TC}$ & TG & LDL-C & Calcified & TGF- $\beta$ & $\mathrm{MCP}-1$ & TLR4 \\
\hline Necrotic volume percentage & $0.793^{\star \star}$ & $0.556^{\star *}$ & $0.751^{\star \star}$ & $0.517^{\star \star}$ & $-0.453^{\star}$ & $0.457^{\star}$ & $0.493^{\star \star}$ \\
\hline
\end{tabular}

*, $\mathrm{P}<0.05$; ${ }^{\star \star}, \mathrm{P}<0.01$. TC, total cholesterol; TG, triglycerides; LDL-C, low-density lipoprotein cholesterol; TGF- $\beta$, transforming growth factor- $\beta$; MCP-1, monocyte chemoattractant protein-1; TLR4, toll-like receptor 4.

observed that LDL-C correlated with the variables derived from IVUS.

\section{Discussion}

The present study demonstrates the efficacy of the PCSK9 inhibitor, evolocumab, in inhibiting the progression and stability of atherosclerotic plaques in rabbits using grayscale IVUS and iMAP-IVUS. Evolocumab was more effective than atorvastatin in reducing the progression and stability of atherosclerotic plaques. The PAV was found to be significantly correlated with the serum levels of TG, TC, LDL-C, MCP-1, and TGF- $\beta$, and the expression of TLR4 in the abdominal aorta.

Previous studies in human subjects have demonstrated that statins can reduce the volume of atherosclerotic plaques (38-41). The GLAGOV (Global Assessment of Plaque Regression with a PCSK9 Antibody as Measured by IVUS) trial demonstrated that PCSK9 inhibitors could effectively reduce the volume of atherosclerotic 
Table 2 Multivariate linear regression analysis results for IVUS variables and respective predictors

\begin{tabular}{lcc}
\hline IVUS variable and respective predictor & Coefficient & $P$ value \\
\hline PAV $\left(\mathrm{mm}^{3}\right)$ & -21.007 & $<0.001$ \\
Ato & -34.46 & 0.004 \\
Evo & 0.264 & 0.05 \\
LDL-C $(\mathrm{mg} / \mathrm{dL})$ & & \\
Fibrotic\% & 2.509 & 0.253 \\
Ato & 12.326 & $<0.001$ \\
Evo & -0.056 & $<0.001$ \\
LDL-C $(\mathrm{mg} / \mathrm{dL})$ & & \\
Necrotic\% & -2.133 & 0.033 \\
Ato & -7.753 & $<0.001$ \\
Evo & 0.041 & $<0.001$ \\
LDL-C $(\mathrm{mg} / \mathrm{dL})$ & & \\
\hline
\end{tabular}

All values are the follow-up values of week 18. IVUS, intravascular ultrasound; PAV, percent atheroma volume; LDL-C, low-density lipoprotein cholesterol. Ato, atorvastatin; Evo, evolocumab.

plaques (42), similar to the results obtained in our study. Previous studies have demonstrated the plaque-stabilizing effects of statins. Using IB-IVUS and optical coherence tomography (OCT), Hattori and colleagues have verified that pitavastatin increases the thickness of fibrous caps and reduces the lipidic\% in human coronary plaques (43). Using OCT, Komukai and colleagues obtained similar results with atorvastatin (44). Although the iMAP-IVUS method differed from IB-IVUS with respect to the imaging mechanism, similar results were obtained in our study due to the fact that the necrotic\% determined with iMAP was positively correlated with the lipid pool calculated using IB-IVUS (45). By employing the iMAP-IVUS method and multivariate linear regression analysis, we additionally observed that evolocumab had a more significant effect on the regression and stabilization of the atherosclerotic plaques than the effect exhibited by atorvastatin, as indicated by the increased fibrotic\% and decreased necrotic\% following evolocumab treatment.

LDL is vital to the formation of atherosclerotic plaques. As inhibitors of 3-hydroxy-3-methylglutaryl coenzyme A, statins are standard anti-atherosclerotic pharmacotherapeutic agents that are used to lower the levels of lipid in the blood. PCSK9 inhibitors can reduce the recycling of the LDL receptor to the hepatic surface, thereby limiting the removal of LDL particles from the circulation (29). As evolocumab is a type of monoclonal antibody against PCSK9, it can notably reduce the levels of LDL-C when administered alone or in combination with statins (46). In the present study, evolocumab was more effective than atorvastatin in reducing the serum levels of TG, TC, and LDL-C. These results are consistent with those of previous studies.

The development of atherosclerosis may have an inflammatory and immune basis (47). TLRs are important innate immune receptors that also function as patternrecognition receptors. Some TLRs, including TLR4, can directly promote atherosclerotic progression via the stimulation of macrophages $(48,49)$. TLR4 is expressed in the arterial walls of normal subjects at low levels, but is overexpressed in atheroma, and is mainly concentrated in the shoulder region where the plaque is most likely to rupture (50). Therefore, TLR4 can be an ideal marker for indicating plaque vulnerability. The progress of atherosclerotic inflammation is mediated by the TLR4nuclear factor- $\kappa \mathrm{B}(\mathrm{NF}-\kappa \mathrm{B})$ pathway, which induces the overexpression of several inflammatory cytokines including MCP-1 (18). MCP-1 recruit monocytes to the vessel wall and is expressed in the macrophage-rich regions of atherosclerotic plaques (51). The proatherosclerotic effects of MCP-1 have been proved by gene deletion experiments (52). The results of the present study indicated that atorvastatin reduced the expression of TLR4 and MCP-1, and this effect was intensified by treatment with evolocumab. These results indicated the anti-inflammatory effect of evolocumab was mediated via the decrease in the expression of MCP-1/TLR4 and an increase in the expression of TGF- $\beta$. These findings provide novel evidence for elucidating the mechanisms underlying the anti-inflammatory effects of evolocumab.

In conclusion, the results of our study suggested that evolocumab probably regulated the levels of lipid and cholesterol via the inflammatory pathways. Additionally, the use of IVUS techniques would help to elucidate the mechanism underlying the inhibition of atherosclerotic plaques by evolocumab.

\section{Acknowledgments}

Funding: This study was supporteded by the National Science Foundation of China (81670245) and Beijing Linghang Fund (LHJJ20159227). 


\section{Footnote}

Reporting Checklist: The authors have completed the ARRIVE reporting checklist. Available at http://dx.doi. org/10.21037/apm-20-690

Data Sharing Statement: Available at http://dx.doi. org/10.21037/apm-20-690

Conflicts of Interest: All authors have completed the ICMJE uniform disclosure form (available at http://dx.doi. org/10.21037/apm-20-690). The authors have no conflicts of interest to declare.

Ethical Statement: The authors are accountable for all aspects of the work in ensuring that questions related to the accuracy or integrity of any part of the work are appropriately investigated and resolved. Experiments were performed under a project license (No. 2016061) granted by the ethics committee of Jinan Central Hospital Affiliated to Shandong University, in compliance with the Guide for the Care and Use of Laboratory Animals published by the US National Institutes of Health (NIH Publication No. 8523, revised 2011).

Open Access Statement: This is an Open Access article distributed in accordance with the Creative Commons Attribution-NonCommercial-NoDerivs 4.0 International License (CC BY-NC-ND 4.0), which permits the noncommercial replication and distribution of the article with the strict proviso that no changes or edits are made and the original work is properly cited (including links to both the formal publication through the relevant DOI and the license). See: https://creativecommons.org/licenses/by-nc-nd/4.0/.

\section{References}

1. Libby P, Ridker PM and Maseri A. Inflammation and Atherosclerosis. Circulation 2002;105:1135-43.

2. Fuster V, Badimon L, Badimon JJ, et al. The Pathogenesis of Coronary Artery Disease and the Acute Coronary Syndromes (2). N Engl J Med 1992;326:310-8.

3. Rubio-Ruiz ME, Peredo-Escárcega AE, Cano-Martínez A, et al. An Evolutionary Perspective of Nutrition and Inflammation as Mechanisms of Cardiovascular Disease. Int J Evol Biol 2015;2015:179791.

4. Gargalovic PS, Imura M, Zhang B, et al. Identification of inflammatory gene modules based on variations of human endothelial cell responses to oxidized lipids. Proc Natl Acad Sci U S A 2006;103:12741-6.

5. Joyce CW, Amar MJ, Lambert G, et al. The ATP binding cassette transporter A1 (ABCA1) modulates the development of aortic atherosclerosis in $\mathrm{C} 57 \mathrm{BL} / 6$ and apoE-knockout mice. Proc Natl Acad Sci U S A 2002;99:407-12.

6. Parissis JT, Adamopoulos S, Venetsanou KF, et al. Serum profiles of $\mathrm{C}-\mathrm{C}$ chemokines in acute myocardial infarction: possible implication in postinfarction left ventricular remodeling. J Interferon Cytokine Res 2002;22:223-9.

7. Gawaz M, Neumann FJ, Dickfeld T, et al. Activated platelets induce monocyte chemotactic protein-1 secretion and surface expression of intercellular adhesion molecule-1 on endothelial cells. Circulation 1998;98:1164-71.

8. Prosser HC, $\mathrm{Ng} \mathrm{MK}$, Bursill CA. The role of cholesterol efflux in mechanisms of endothelial protection by HDL. Curr Opin Lipidol 2012;23:182-9.

9. Ma Y, Yabluchanskiy A, Hall ME, et al. Using plasma matrix metalloproteinase-9 and monocyte chemoattractant protein-1 to predict future cardiovascular events in subjects with carotid atherosclerosis. Atherosclerosis 2014;232:231-3.

10. Ding SF, Zhang Y, Zhang $M$, et al. The role of atherosclerotic plaque stability and inflammation in the pathogenesis of acute coronary syndrome. Zhonghua Xin Xue Guan Bing Za Zhi 2006;34:512-4.

11. Hartford M, Wiklund O, Mattsson Hulten L, et al. CRP, interleukin-6, secretory phospholipase A2 group IIA, and intercellular adhesion molecule-1 during the early phase of acute coronary syndromes and long-term follow-up. Int J Cardiol 2006;108:55-62.

12. Frutkin AD, Otsuka G, Stempien-Otero A, et al. TGF[beta] 1 limits plaque growth, stabilizes plaque structure, and prevents aortic dilation in apolipoprotein E-null mice. Arterioscler Thromb Vasc Biol 2009;29:1251-7.

13. Ruiz-Ortega M, Rodriguez-Vita J, Sanchez-Lopez E, et al. TGF-beta signaling in vascular fibrosis. Cardiovasc Res 2007;74:196-206.

14. Pardali E, Ten Dijke P. TGFbeta signaling and cardiovascular diseases. Int J Biol Sci 2012;8:195-213.

15. Roshan MH, Tambo A, Pace NP. The Role of TLR2, TLR4, and TLR9 in the Pathogenesis of Atherosclerosis. Int J Inflam 2016;2016:1532832.

16. $\mathrm{Xu} \mathrm{XH,} \mathrm{Shah} \mathrm{PK,} \mathrm{Faure} \mathrm{E,} \mathrm{et} \mathrm{al.} \mathrm{Toll-like} \mathrm{receptor-4} \mathrm{is}$ expressed by macrophages in murine and human lipid-rich atherosclerotic plaques and upregulated by oxidized LDL. Circulation 2001;104:3103-8. 
17. Edfeldt K, Swedenborg J, Hansson GK, et al. Expression of toll-like receptors in human atherosclerotic lesions: a possible pathway for plaque activation. Circulation 2002;105:1158-61.

18. Vink A, Schoneveld AH, van der Meer JJ, et al. In vivo evidence for a role of toll-like receptor 4 in the development of intimal lesions. Circulation 2002;106:1985-90.

19. Pasterkamp G, Van Keulen JK, De Kleijn DP. Role of Toll-like receptor 4 in the initiation and progression of atherosclerotic disease. Eur J Clin Invest 2004;34:328-34.

20. den Dekker WK, Cheng C, Pasterkamp G, et al. Toll like receptor 4 in atherosclerosis and plaque destabilization. Atherosclerosis 2010;209:314-20.

21. Suzuki H, Kobayashi H, Sato F, Yonemitsu Y, et al. Plaquestabilizing effect of pitavastatin in Watanabe heritable hyperlipidemic (WHHL) rabbits. J Atheroscler Thromb 2003;10:109-16.

22. Shiomi M, Yamada S, Ito T. Atheroma stabilizing effects of simvastatin due to depression of macrophages or lipid accumulation in the atheromatous plaques of coronary plaque-prone WHHL rabbits. Atherosclerosis 2005;178:287-94.

23. Tian J, Hu S, Sun Y, et al. Vasa vasorum and plaque progression, and responses to atorvastatin in a rabbit model of atherosclerosis: contrast-enhanced ultrasound imaging and intravascular ultrasound study. Heart 2013;99:48-54.

24. Li Z, Wang L, Hu X, et al. Effect of rosuvastatin on atherosclerotic plaque stability:An intravascular ultrasound elastography study. Atherosclerosis 2016;248:27-35.

25. Shin ES, Garcia-Garcia HM, Ligthart JM, et al. In vivo findings of tissue characteristics using iMap IVUS and Virtual Histology IVUS. EuroIntervention 2011;6:1017-9.

26. Sathyanarayana S, Carlier S, Li W, et al. Characterisation of atherosclerotic plaque by spectral similarity of radiofrequency intravascular ultrasound signals. EuroIntervention 2009;5:133-9.

27. Liu J, Wang Z, Wang WM, et al. Feasibility of diagnosing unstable plaque in patients with acute coronary syndrome using iMap-IVUS. J Zhejiang Univ Sci B 2015;16:924-30.

28. Koga S, Ikeda S, Miura M, et al. iMap-Intravascular Ultrasound Radiofrequency Signal Analysis Reflects Plaque Components of Optical Coherence TomographyDerived Thin-Cap Fibroatheroma. Circ J 2015;79:2231-7.

29. Abifadel M, Varret M, Rabes JP, et al. Mutations in PCSK9 cause autosomal dominant hypercholesterolemia. Nat Genet 2003;34:154-6.

30. Roth EM, McKenney JM, Hanotin C, et al. Atorvastatin with or without an antibody to PCSK9 in primary hypercholesterolemia. N Engl J Med 2012;367:1891-900.

31. Lee P, Hegele RA. Current Phase II proprotein convertase subtilisin/kexin 9 inhibitor therapies for dyslipidemia. Expert Opin Investig Drugs 2013;22:1411-23.

32. Poirier S, Mayer G. The biology of PCSK9 from the endoplasmic reticulum to lysosomes:new and emerging therapeutics to control low-density lipoprotein cholesterol. Drug Des Devel Ther 2013;7:1135-48.

33. Chen WQ, Zhang L, Liu YF, et al. Prediction of atherosclerotic plaque ruptures with high-frequency ultrasound imaging and serum inflammatory markers. Am J Physiol Heart Circ Physiol 2007;293:H2836-44.

34. Zhang L, Liu Y, Zhang PF, et al. Peak radial and circumferential strain measured by velocity vector imaging is a novel index for detecting vulnerable plaques in a rabbit model of atherosclerosis. Atherosclerosis 2010;211:146-52.

35. Zhang L, Liu Y, Lu XT, et al. Intraplaque injection of Ad5-CMV.p53 aggravates local inflammation and leads to plaque instability in rabbits. J Cell Mol Med 2009; 13:2713-23.

36. Mintz GS, Nissen SE, Anderson WD, et al. American College of Cardiology Clinical Expert Consensus Document on Standards for Acquisition, Measurement and Reporting of Intravascular Ultrasound Studies (IVUS). A report of the American College of Cardiology Task Force on Clinical Expert Consensus Documents. J Am Coll Cardiol 2001;37:1478-92.

37. Nicholls SJ, Hsu A, Wolski K, et al. Intravascular ultrasound-derived measures of coronary atherosclerotic plaque burden and clinical outcome. J Am Coll Cardiol 2010;55:2399-407.

38. Hiro T, Kimura T, Morimoto T, et al. Effect of intensive statin therapy on regression of coronary atherosclerosis in patients with acute coronary syndrome:a multicenter randomized trial evaluated by volumetric intravascular ultrasound using pitavastatin versus atorvastatin (JAPANACS [Japan assessment of pitavastatin and atorvastatin in acute coronary syndrome] study). J Am Coll Cardiol 2009;54:293-302.

39. Ballantyne CM, Raichlen JS, Nicholls SJ, et al. Effect of rosuvastatin therapy on coronary artery stenoses assessed by quantitative coronary angiography: a study to evaluate the effect of rosuvastatin on intravascular ultrasound-derived coronary atheroma burden. Circulation 2008;117:2458-66.

40. Nissen SE, Tuzcu EM, Schoenhagen P, et al. Effect of intensive compared with moderate lipid-lowering therapy 
on progression of coronary atherosclerosis:a randomized controlled trial. Jama 2004;291:1071-80.

41. Takayama T, Hiro T, Yamagishi M, et al. Effect of rosuvastatin on coronary atheroma in stable coronary artery disease: multicenter coronary atherosclerosis study measuring effects of rosuvastatin using intravascular ultrasound in Japanese subjects (COSMOS). Circ J 2009;73:2110-7.

42. Nicholls SJ, Puri R, Anderson T, et al. Effect of Evolocumab on Progression of Coronary Disease in Statin-Treated Patients: The GLAGOV Randomized Clinical Trial. JAMA 2016;316:2373-84.

43. Hattori K, Ozaki Y, Ismail TF, et al. Impact of statin therapy on plaque characteristics as assessed by serial OCT, grayscale and integrated backscatter-IVUS. JACC Cardiovasc Imaging 2012;5:169-77.

44. Komukai K, Kubo T, Kitabata H, et al. Effect of atorvastatin therapy on fibrous cap thickness in coronary atherosclerotic plaque as assessed by optical coherence tomography: the EASY-FIT study. J Am Coll Cardiol 2014;64:2207-17.

45. Yamada R, Okura H, Kume T, et al. A comparison between $40 \mathrm{MHz}$ intravascular ultrasound iMap imaging system and integrated backscatter intravascular ultrasound. J Cardiol 2013;61:149-54.

46. Robinson JG, Nedergaard BS, Rogers WJ, et al. Effect

Cite this article as: Kong Q, Liu M, Li Y, Zhu Q, Su G. Effect of evolocumab on the progression and stability of atherosclerotic plaques as evaluated by grayscale and iMAP-IVUS. Ann Palliat Med 2020;9(5):3078-3088. doi: 10.21037/apm-20-690 of evolocumab or ezetimibe added to moderate- or highintensity statin therapy on LDL-C lowering in patients with hypercholesterolemia: the LAPLACE-2 randomized clinical trial. JAMA 2014;311:1870-82.

47. Libby P. Inflammation in atherosclerosis. Nature 2002;420:868-74.

48. Lee JG, Lim EJ, Park DW, et al. A combination of Lox-1 and Nox1 regulates TLR9-mediated foam cell formation. Cell Signal 2008;20:2266-75.

49. Katoh S, Honda S, Watanabe T, et al. Atrial endothelial impairment through Toll-like receptor 4 signaling causes atrial thrombogenesis. Heart Vessels 2014;29:263-72.

50. Holloway JW, Yang IA, Ye S. Variation in the toll-like receptor 4 gene and susceptibility to myocardial infarction. Pharmacogenet Genomics 2005;15:15-21.

51. Namiki M, Kawashima S, Yamashita T, et al. Local overexpression of monocyte chemoattractant protein-1 at vessel wall induces infiltration of macrophages and formation of atherosclerotic lesion: synergism with hypercholesterolemia. Arterioscler Thromb Vasc Biol 2002;22:115-20.

52. Inoue S, Egashira K, Ni W, et al. Anti-monocyte chemoattractant protein-1 gene therapy limits progression and destabilization of established atherosclerosis in apolipoprotein E-knockout mice. Circulation 2002;106:2700-6. 

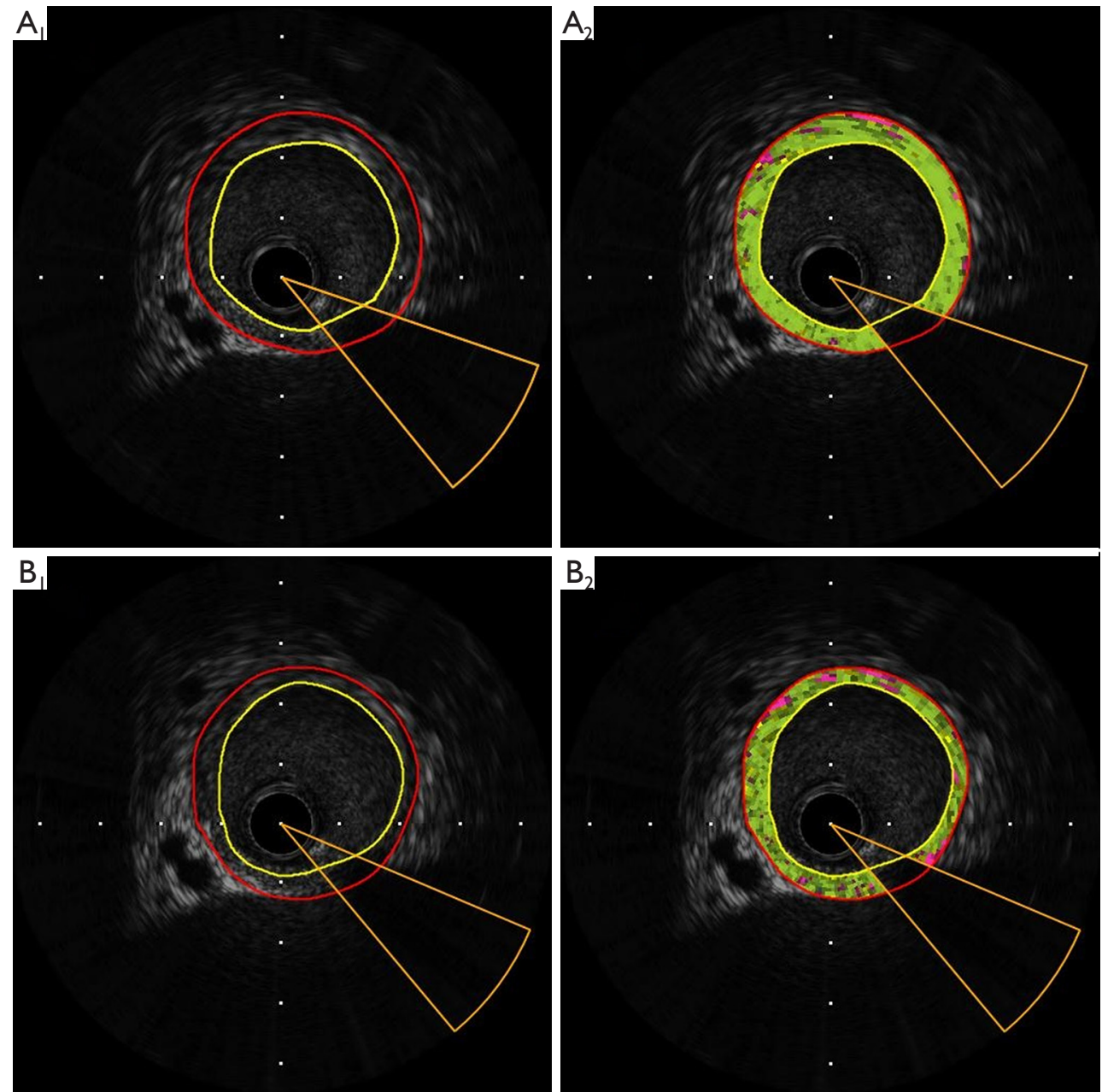

Figure S1 Representative grayscale and corresponding iMAP-IVUS images. (A1) Representative grayscale IVUS transverse image of a section of the abdominal aorta of a rabbit in the Evo group at 10 weeks. (A2) Corresponding iMAP-IVUS image for A1. (B1) Grayscale IVUS transverse image of the same section in A1 at 18 weeks. (B2) Corresponding iMAP-IVUS image for B1. The four tissue compositions are depicted in light green (fibrotic), yellow (lipidic), pink (necrotic), and blue (calcific). The cross-sectional area of the plaque in A1 is 4.68 mm ${ }^{2}$, while the area of the plaque in $\mathrm{B} 1$ is $3.59 \mathrm{~mm}^{2}$. 